\title{
Influence of Biochar Particulate on the Sorption Behaviour of Sansevieria Cylindrica Reinforced Vinyl Ester Composite
}

\author{
R. Deepak Joel Johnson, V. Arumugaprabu, P. Sivaranjana
}

\begin{abstract}
Purpose of this research is to evaluate the influence of biochar from corn cob and wood waste residues on the water resistivity of the Sansevieria cylindrica reinforced vinyl ester composite (SCVEC) to be utilized in structural and marine applications. Biochar filled SCVEC was allowed to immerse in different sources of waters like seawater, ground water and distilled water to study the water absorption of the material. Kinetics of water molecule transferring into the composite during immersion was studied by determining the diffusion coefficient, permeability coefficient and sorption coefficient. Fabricated composites were analysed for its Fickian behaviour under 720 Hrs of immersion in different water medium. Chemical resistivity for the fabricated composite were also studied to make the composite more accessible to marine environment. Results reveals that increase in biochar loading up to certain wt. \% increases the water resistivity of the composite. Reinforcement of Biochar derived from corn cob was found to have potential water resistivity property compared to the biochar derived from wood wastes.
\end{abstract}

Keywords: Sorption Behaviour, Chemical resistivity, Sansevieria cylindrica, Biochar, Vinyl ester, Fickian Behaviour.

\section{INTRODUCTION}

$\mathrm{N}_{\mathrm{a}}$ its way in severing as potential replacement of plastics in sectors like automobile, aircraft and construction industries due to its high strength to weight ratio. But researchers find it challenging to replace these kinds of NFRP composite to use in marine application due to the hydrophilic nature of the natural fibre. Our research aims to fabricate a cost-effective water resistive composite to be utilized in marine applications. The problem with biodegradability leads to the usage of agricultural and forestry wastes in making pioneering products. Researchers around the globe knows the issues and explored their idea towards fabricating composites from wastes [1]. As a secondary reinforcing material i.e. fillers, carbon material receipts a great consideration due to their

Revised Manuscript Received on December 17, 2019.

R. Deepak Joel Johnson, Department of Mechanical Engineering, Saveetha School of Engineering, Saveetha Institute of Medical and Technical Sciences, Saveetha Nagar, Thandalam, Chennai -602 105, Tamil Nadu, India. Email: rdeepakjoeljohnson@gmail.com

V. Arumugaprabu*, School of Automotive and Mechanical Engineering, Kalasalingam Academy of Research and Education, Srivilliputhur - 626 126, Tamil Nadu, India. Email: v.arumugaprabu@klu.ac.in

P. Sivaranjana*, Department of Chemistry, Kalasalingam Academy of Research and Education, Srivilliputhur - 626 126, Tamil Nadu, India. Email: p.sivaranjana@klu.ac.in. ability to boost the mechanical, electrical and thermal properties of the composite using carbon as filler material [2]. The main drawback of found by many researchers by utilizing this carbon filler material is its cost. For larger scale production of composite when used carbon as filler material the final cost of the fabricated composite is extensively high. Researcher were looking for a promising alternate with high carbon content. Also, researchers exploring the possibility of utilizing the waste materials as possible source to produce these carbon fillers. One such material derived from the waste biomasses by pyrolysis process is called Biochar [3]. Biochar, attained by the pyrolysis of biowaste, has demonstrated as potential replacement to expensive carbon fillers as of its capability to advance the composites mechanical, electrical and thermal properties [4]. The absorbed water not only depreciates the mechanical and thermal properties, but also induce corrosion on the surface of the material which destroys the adhesion between the binder and the enhanced materials. Thus, it proves the necessity of studying water resistivity of any fabricated composite [5]. Biochar produced from pine cone, wood shaving and plastic waste were reinforced with epoxy resin and the water absorption studies were done. It was inferred that pine cone char composite and Plastic waste char composite showed less water diffusivity compared to that of wood shaving char composite [6]. Biochar derived from miscanthus, switchgrass and softwood chip were studied for its water absorption capacity. Results revealed that Biochar having more oxygenated groups will have more water absorption since the water molecule comes into contact firstly with the oxygenated surface through hydrogen bonding. Also, the study reveals that size of the pores in the biochar structure plays a vital role in water resistivity of the Biochar material [7]. Water diffusivity of the composite was carried in three different water sources namely sea water, distilled water and ground/ fresh water. It is found that in seawater due to the presence of salt molecules, diffusion rate gets slower compare to other water sources [8-10]. To evaluate the capability of the water that infiltrate the fabricated composite, the diffusion coefficient was determined using the Fickian equation, from which the behavior of the composite for its water resistivity is studied [11-13]. This research concentrates in studying the influence of Biochar particle produced from corn cob and wood waste residues on the water resistivity of the Sansevieria cylindrica reinforced vinyl ester composite. 


\section{MATERIALS AND METHODS}

\section{A. Materials Used}

Sansevieria cylindrica fibre (SCF) was the primary reinforcing material and Biochar derived from corn cob and wood waste residue being the secondary reinforcement material. Corn cob was collected from the nearby village of the Rajapalayam whereas the wood waste was collected from the saw mill industries in Rajapalayam, Virudhunagar, India. Vinyl ester resin purchased from Vasavibala private Ltd., Chennai, India with the trade name VBR 2301 was used as the matrix material. Seawater was taken from the Bay of Bengal, Distilled water was purchased from a private vendor and groundwater was taken from the Kalasalingam Academy of
Research Education campus, India. The $\mathrm{pH}$ values of all the seawater, groundwater and distilled water were measured using $\mathrm{pH}$ strips and the values are tabulated in Table 1.

\section{B. Composite Fabrication}

Biochar filled SCVEC material was fabricated using compression molding method with pressure $120 \mathrm{Kgf} / \mathrm{cm}^{2}$ and with the dimension of composite plate $300 \times 130 \times 3 \mathrm{~mm}$. Prior to the reinforcement SCF was chemically treated with $\mathrm{NaOH}$ solution. Fabricated Biochar filled SCVEC material was cut as per ASTM D570 standards to accomplish water diffusivity test. Table 2 shows the designation of the fabricated Biochar filled SCVEC materials. Table 1 shows the fabricated composites and its designation.

Table 1: Composite designation for the Biochar filled SCVEC.

\begin{tabular}{cc}
\hline Composite Details & Composite Designation \\
\hline 0 wt. \% Corncob Biochar filled SCVEC & 0 wt. \% CBC \\
2 wt. \% Corncob Biochar filled SCVEC & 2 wt. \% CBC \\
4 wt. \% Corncob Biochar filled SCVEC & 4 wt. \% CBC \\
6 wt. \% Corncob Biochar filled SCVEC & 6 wt. \% CBC \\
8 wt. \% Corncob Biochar filled SCVEC & 8 wt. \% CBC \\
10 wt. \% Corncob Biochar filled SCVEC & 10 wt. \% CBC \\
0 wt. \% Wood waste Biochar filled SCVEC & 0 wt. \% WBC \\
2 wt. \% Wood waste Biochar filled SCVEC & 2 wt. \% WBC \\
4 wt. \% Wood waste Biochar filled SCVEC & 4 wt. \% WBC \\
6 wt. \% Wood waste Biochar filled SCVEC & 6 wt. \% WBC \\
8 wt. \% Wood waste Biochar filled SCVEC & 8 wt. \% WBC \\
10 wt. \% Wood waste Biochar filled SCVEC & $10 \mathrm{wt} \%$ WBC \\
\hline
\end{tabular}

\section{Moisture diffusion behaviour}

Moisture diffusivity of the Biochar filled SCVEC material was conducted as per ASTM D570 and initial weight of the specimen in dry condition was noted. Then, the samples were allowed to immerse in seawater, groundwater and distilled water at room temperature for $720 \mathrm{hrs}$. The samples were weighted for every 24 hours after cleaning out the water on the specimen surface using filter papers. Precision of $0.001 \mathrm{~g}$ was maintained to measure the weight of the specimen. Percentage of moisture diffusivity was calculated using equation (3).

$$
\% \text { Moisure diffusion }=\frac{\mathrm{W}_{\mathrm{t}}-\mathrm{W}_{\text {Initial }}}{\mathrm{W}_{\text {Initial }}} \times 100
$$

Where,

$\mathrm{W}_{\mathrm{t}}$ and $\mathrm{W}_{\text {Initial }}$ are the weight of the sample at time $\mathrm{t}$ and the initial weight of the sample.

To perceive the kinetics of the specimen under immersion study, diffusion coefficient (D) one of the decisive constraints in Fick's model were measured using equation (4), which is used to study the ability of the water molecules to intrude into the composite.

$$
\mathrm{D}=\pi\left[\frac{\theta \mathrm{h}}{4 \mathrm{M}_{\mathrm{m}}}\right]^{2}
$$

$\theta$ is the slope of the linear part of $M_{t}$ against the $V_{t}$ curve, $h$ is the thickness of the specimen and $\mathrm{M}_{\mathrm{m}}$ is the water diffusion percentage at an infinite time. Sorption behavior of the Biochar filled composite at the equilibrium condition was determined with the sorption coefficient (S) which was calculated using equation (5).

$$
\mathrm{S}=\frac{\mathrm{M}_{\mathrm{m}}}{\mathrm{M}_{\mathrm{t}}}
$$

Using the sorption coefficient and diffusion coefficient, permeability coefficient $(\mathrm{P})$ of the composite, i.e., the net effect of sorption and diffusion behavior of the composite after prolonged water uptake can be calculated using equation (6).

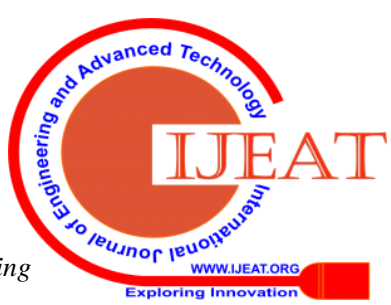




$$
\mathrm{P}=\mathrm{D} \times \mathrm{S}
$$

To pursue the sorption mechanism of the SCVEC material with Fick's theory, the water uptake of the Biochar filled SCVEC material was fitted to equation (7),

$$
\log \left(\frac{\mathrm{M}_{\mathrm{t}}}{\mathrm{M}_{\mathrm{m}}}\right)=\log k+n \log t
$$

Where $M_{t}$ and $M_{m}$ are the moisture diffusion percentage at time $\mathrm{t}$ and equilibrium, $\mathrm{k}$ is a constant term which describes the interaction between the specimen and the water whereas $n$ denotes the sorption mechanism of the biochar filled composite specimen.

\section{Chemical Resistivity Test}

Chemical resistance on the Biochar filled Sansevieria cylindrica reinforced vinyl ester was conducted as per ASTM D 543-87. The Influence of Biochar loading on SCVEC material were evaluated for its resistivity to the solvents, acids and alkali's by immersing the composite specimen in toluene, benzene, nitric acid, hydrochloric acid, $\mathrm{NaOH}$ and $\mathrm{Na}_{2} \mathrm{CO}_{3}$. Initial weight of the specimen in dry condition is weighted using physical balance with $0.001 \mathrm{~g}$ precision and then the specimens were allowed to immerge in the respective chemical reagents for $24 \mathrm{Hrs}$. Immediately after removal of specimens from chemical immersion, it is washed in distilled water and dried by pressing them with a filter paper at room temperature. The samples were then weighed and the percentage weight loss/gain was determined using the equation (5).

\section{RESULTS AND DISCUSSION}

\section{A. Moisture diffusivity of Biochar filled composite}

Moisture diffusion of the biochar filled SCVEC material was studied by immersing specimen into various medium of water sources like seawater, groundwater and distilled water. Fig. 1 shows the specimen immersed in seawater for 720 hours. It is observed that for corn cob biochar filled SCVEC specimens the water resistivity increases as the biochar wt. \% increases till 6 wt. \% beyond which water resistivity decreases. But in case of wood waste biochar filled composites the water resistivity decreases as the biochar content increases. It is because of the huge number of pores present in the wood waste biochar.

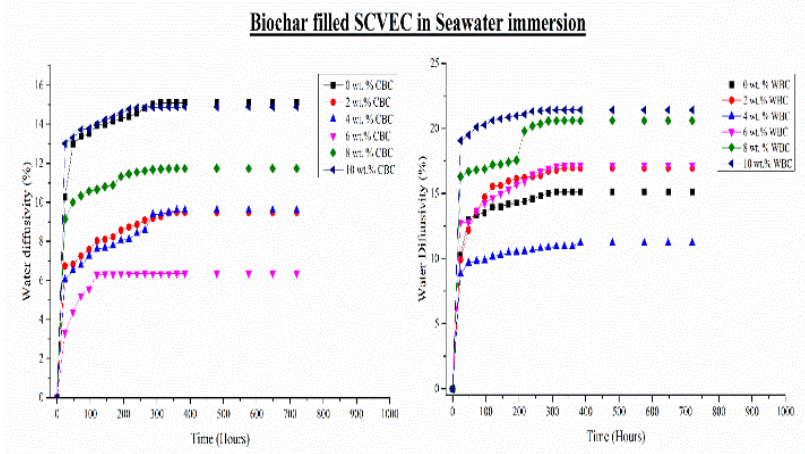

Fig. 1: Biochar filled Sansevieria cylindrica reinforced Vinyl ester composite in seawater immersion

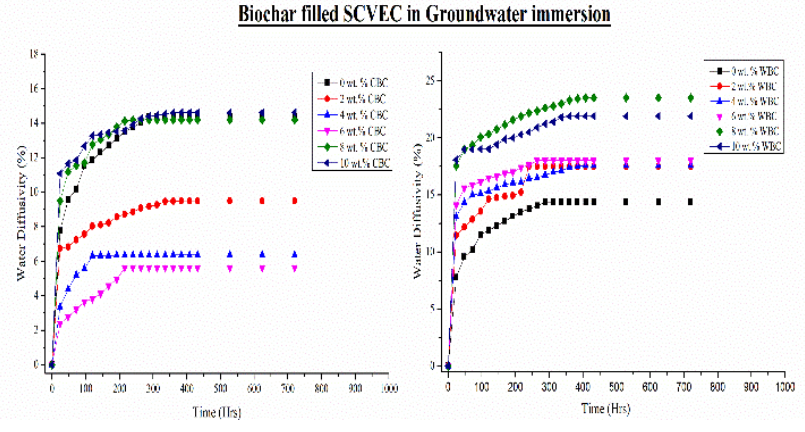

Fig. 2: Biochar filled Sansevieria cylindrica reinforced Vinyl ester composite in Ground water immersion

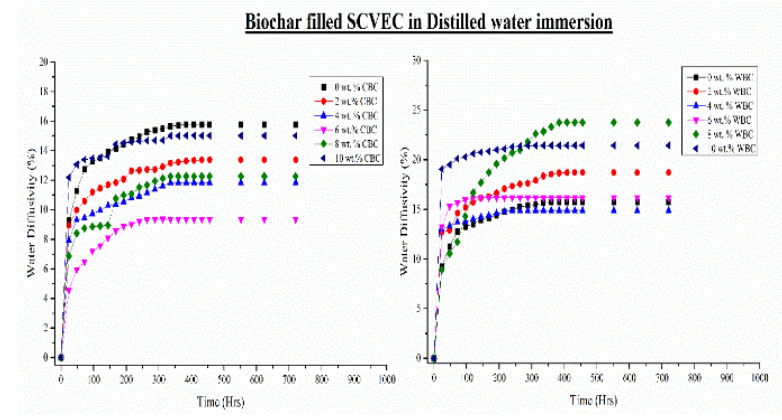

Fig. 3: Biochar filled Sansevieria cylindrica reinforced Vinyl ester composite in Distilled water immersion

Fig. 2 shows the biochar filled SCVEC material under 720 hours of immersion in ground water. It was inferred from the graphs that corncob biochar filled SCVEC showed better water resistivity compared to wood waste biochar filled SCVEC. Also, it is observed that corncob biochar up to $6 \mathrm{wt}$. $\%$ can only hold the resistivity of the composite beyond $6 \mathrm{wt}$. $\%$ the water absorption in the specimen gets increased.

It is observed that a similar trend was followed when the biochar filled SCVEC material immersed in distilled water as it is in other two medium. Water resistivity of $6 \mathrm{wt}$. \% corncob filled SCVEC material was having the better values compared to all other combinations. Correspondingly $6 \mathrm{wt}$. \% corncob Biochar filled SCVEC has good resistive capacity when immersed in seawater compared to other two mediums. It is due to the salt content present in sea water which restrict the free transport of water molecules infiltrating the composite surface. Irrespective to the immersion medium corncob biochar filled SCVEC showed better water resistivity compared to wood waste biochar filled SCVEC.

\section{B. Kinematics of water infiltrate into the Biochar filled composite}

To determine the significance of water infiltrating the composite material, three distinct parameters were calculated namely Diffusion coefficient, Permeability coefficient and Sorption coefficient. Table 2 shows the values of diffusion coefficient, permeability coefficient and sorption coefficient of specimens immersed in seawater, ground water and distilled water. 
Higher values of diffusion coefficient represent better water resistivity of the composite under immersion. It is noted from the table that diffusion coefficient irrespective to the immersion source increases as the corncob biochar wt. \% increases till $6 \mathrm{wt}$ \% after that it tends to decrease drastically. In case of wood waste biochar filled composite, the diffusion coefficient increases as the biochar wt. \% increase, resulting in poor water resistivity regardless to the water source for immersion. Diffusion coefficient and permeability coefficient values states the 6 wt. \% corncob biochar filled SCVEC have the capability to resist water effectively compared to other wt. $\%$ of Biochar filled composites.

Table 2: Diffusion coefficient $\left(D \times 10^{-12}\right)$, Permeability coefficient $\left(P \times 10^{-12}\right)$ and sorption coefficient of Biochar filled SCVEC in Seawater, Ground water and Distilled water.

\begin{tabular}{l|ccc|ccc|ccc}
\hline \multirow{2}{*}{$\begin{array}{c}\text { Composite } \\
\text { Designation }\end{array}$} & \multicolumn{3}{|c|}{ Sea water } & \multicolumn{3}{c|}{ Ground Water } & \multicolumn{3}{c}{ Distilled Water } \\
\cline { 2 - 10 } & S & D & P & S & D & P & S & D & P \\
\hline 0 wt. \% CBC & 1.47 & 1.53 & 2.25 & 1.85 & 4.35 & 8.05 & 1.70 & 1.67 & 2.84 \\
2 wt.\% CBC & 1.41 & 1.59 & 2.24 & 1.41 & 0.98 & 1.38 & 1.50 & 2.08 & 3.11 \\
4 wt. \% CBC & 1.59 & 2.05 & 3.25 & 1.90 & 4.43 & 8.42 & 1.49 & 2.65 & 3.95 \\
6 wt.\% CBC & 1.90 & 4.52 & 8.59 & 2.36 & 13.12 & 30.91 & 2.05 & 3.93 & 8.06 \\
8 wt. \% CBC & 1.28 & 0.59 & 0.75 & 1.49 & 3.53 & 5.28 & 1.78 & 2.89 & 5.14 \\
10 wt.\% CBC & 1.14 & 0.30 & 0.34 & 1.32 & 0.70 & 0.92 & 1.23 & 0.53 & 0.66 \\
2 wt.\% WBC & 1.71 & 1.70 & 2.90 & 1.53 & 2.27 & 3.47 & 1.47 & 0.70 & 1.04 \\
4 wt. \% WBC & 1.27 & 0.44 & 0.56 & 1.34 & 0.53 & 0.71 & 1.14 & 0.08 & 0.09 \\
6 wt.\% WBC & 1.34 & 0.79 & 1.07 & 1.28 & 0.89 & 1.14 & 1.22 & 4.57 & 5.57 \\
8 wt. \% WBC & 1.26 & 0.43 & 0.54 & 1.34 & 0.53 & 0.71 & 2.66 & 3.84 & 10.23 \\
10 wt.\% WBC & 1.12 & 0.23 & 0.26 & 1.21 & 0.37 & 0.45 & 1.12 & 0.23 & 0.26 \\
\hline
\end{tabular}

\section{Chemical resistivity of the Biochar filled composite}

Chemical resistance tests are used to find the ability of a composite to withstand exposure to acids, alkalis, solvents and other chemicals. The chemical resistance tests of these Biochar filled composites were performed in order to find out whether these composites can be used for production of component which can resist chemicals. The weight loss/gain for Biochar filled SCVEC material for acids, solvents and alkaline were shown in Table 2. From Table 2 it is seen that in almost every cases weight gain is observed. Results of the chemical resistance test infers that there is no weight loss in almost every composite which makes the fabricated composite to be utilized in chemical vibrant environments., and therefore it does not seem as if any erosion occurred.

Table 3: Chemical resistance of Biochar filled SCVEC in various chemical reagents

\begin{tabular}{|c|c|c|c|c|c|c|}
\hline \multirow{2}{*}{$\begin{array}{c}\text { Composite } \\
\text { Designation }\end{array}$} & \multicolumn{2}{|c|}{ Acids } & \multicolumn{2}{|c|}{ Alkaline } & \multicolumn{2}{|c|}{ Solvents } \\
\hline & $\mathrm{HCl}$ & $\mathrm{HNO}_{3}$ & $\mathrm{NaOH}$ & $\mathrm{Na}_{2} \mathrm{CO}_{3}$ & Toluene & Benzene \\
\hline 0 wt. $\%$ CBC & 2.02 & 0.15 & 0.26 & 0.25 & 0.00 & 0.00 \\
\hline 2 wt. $\%$ CBC & 0.86 & 0.27 & 0.36 & 0.16 & 0.02 & 0.03 \\
\hline 4 wt. \% CBC & 0.20 & 0.16 & 0.23 & 0.06 & 0.01 & 0.01 \\
\hline 6 wt. $\%$ CBC & 1.05 & 0.07 & 0.27 & 0.07 & 0.02 & 0.02 \\
\hline 8 wt. $\%$ CBC & 13.59 & 0.14 & 0.38 & 0.06 & 0.00 & 0.01 \\
\hline 10 wt. \% CBC & 1.01 & 0.06 & 0.09 & 0.08 & 0.02 & 0.02 \\
\hline 2 wt. $\%$ WBC & 1.55 & 0.24 & 0.26 & 0.07 & 0.02 & 0.02 \\
\hline 4 wt. $\%$ WBC & 2.07 & 0.09 & 0.13 & 0.17 & 0.02 & 0.01 \\
\hline 6 wt. $\%$ WBC & 24.50 & 0.15 & 0.22 & 0.10 & 0.01 & 0.00 \\
\hline 8 wt. $\%$ WBC & 1.67 & 0.16 & 0.24 & 0.12 & 0.04 & 0.02 \\
\hline 10 wt. $\% \mathrm{WBC}$ & 4.99 & 0.21 & 0.28 & 0.20 & 0.01 & \\
\hline
\end{tabular}




\section{CONCLUSIONS}

Conclusion made from the detailed analysis on the water and chemical resistivity of the Biochar filled Sansevieria cylindrica reinforced vinyl ester composite are as follows:

* Irrespective of the immersion medium water resistivity of corncob Biochar filled SCVEC material has less water diffusivity compared to wood waster Biochar filled SCVEC.

* Diffusion coefficient and permeability coefficient increases as the corncob biochar loading till 6 wt. \% after that both these parameters starts falling due to excess concentration of biochar starts to absorb more water.

* Due to the presence of salt content in the seawater sorption coefficient is considerably less in seawater immersion for almost all type of biochar filling. Salts present in seawater disables the easy infiltration of water molecules into the composite.

* Inferred from the chemical resistance test, result reveals that almost every fabricated composite shows good resistance to all the solvents, acids and alkaline up on immersion of the specimen for 24 hours in respectively chemicals.

\section{REFERENCES}

1. O. Das, A. K. Sarmah \& D. Bhattacharyya, "A sustainable and resilient approach through biochar addition in wood polymer composites," Science of the Total Environment, vol. 512, 2015, pp. 326-336.

2. F. Qin and C. Brosseau, "A review and analysis of microwave absorption in polymer composites filled with carbonaceous particles," Journal of applied physics, vol. 111(6), 2012, pp. 4.

3. S. Nanda, A. K. Dalai, F. Berruti and J. A. Kozinski, "Biochar as an Exceptional Bioresource for Energy Carbon Sequestration Agronomy Activated Carbon and Speciality Materials," Waste Biomass Valor, 2015, pp. 1-35.

4. S. Ikram, O. Das and D. Bhattacharyya, "A parametric study of mechanical and flammability properties of biochar reinforced polypropylene composites," Composites Part A: Applied Science and Manufacturing, vol. 91, 2016, pp.177-188.

5. S. Y. Zhang, Y. F. Ding, S. J. Li, X. W. Luo and W. F. Zhou, "Effect of polymeric structure on the corrosion protection of epoxy coatings," Corrosion Science, vol. 44(4), 2002, pp. 861-869.

6. G. Ahmetli, S. Kocaman, I. Ozaytekin and P. Bozkurt, "Epoxy composites based on inexpensive char filler obtained from plastic waste and natural resources," Polymer Composites, vol. 34(4), 2013 , pp. 500-509.

7. E. Behazin, E. Ogunsona, A. Rodriguez-Uribe, A. K. Mohanty, M. Misra and A. O. Anyia, "Mechanical, chemical, and physical properties of wood and perennial grass biochars for possible composite application," BioResources, vol. 11(1), 2015, pp. 1334-1348.

8. R. S. Chen, A. Ghani, M. Hafizuddin, M. N. Salleh, S. Ahmad and M. A. Tarawneh, "Mechanical, water absorption, and morphology of recycled polymer blend rice husk flour biocomposites," J. Appl. Polym. Sci, 2015, pp. 132. https://doi.org/10.1002/app.41494

9. M. Chandrasekar, M. R. Ishak, S. M. Sapuan, Z. Leman and M. Jawaid, "A review on the characterisation of natural fibres and their composites after alkali treatment and water absorption," Plastics Rubber and Composites, vol. 46, 2017, pp. 119 https://doi.org/10.1080/14658011.2017.1298550

10. P. Davies, F. Mazeas and P. Casari, "Sea water aging of glass reinforced composites: shear behaviour and damage modelling," Journal of Composite materials, vol. 35, 2001, pp. 1343. https://doi.org/10.1106/MNBC-81UB-NF5H-P3ML
11. M. K. Gupta and R. K. Srivastava, "Mechanical, thermal and water absorption properties of hybrid sisal/jute fibre reinforced polymer composite," Indian Journal of Engineering Material Science, vol. 23, 2016, pp. 231.

12. S. Joannès, L. Mazé and A. R. Bunsell, "A concentration-dependent diffusion coefficient model for water sorption in composite," Composite Structure, vol. 108, 2014, pp. 111. https://doi.org/10.1016/j.compstruct.2013.09.007

13. H. N. Dhakal, Z. Y. Zhang and M. O. Richardson, "Effect of water absorption on the mechanical properties of hemp fibre reinforced unsaturated polyester composites," Composite Science and

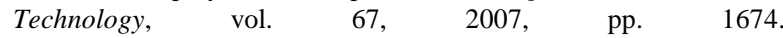
https://doi.org/10.1016/j.compscitech.2006.06.019

\section{AUTHORS PROFILE}

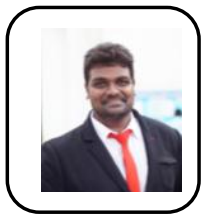

R. Deepak Joel Johnson has perused Bachelor's degree in Mechanical Engineering and Master of Technology in Mechanical Engineering with Manufacturing Engineering as Specialization from Karunya University, India. With two years of experience as Assistant Professor in M. Kumarasamy College of Engineering, Karur and he has completed his $\mathrm{PhD}$ in the field of Polymer Composite at Kalasalingam University, India in the year 2019. Currently he is working as Assistant Professor, Department of Mechanical Engineering, Saveetha School of Engineering, SIMATS Chennai, India. He has more than 15 International Publication in reputed Journals. His areas of interests are Polymer composites, Bio composites, Optimization techniques, Cutting Fluid Application, Composite machining.

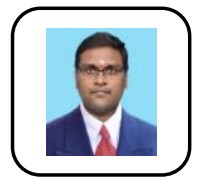

V. Arumugaprabu works as associate professor in the Department of Mechanical Engineering, School of Automotive and Mechanical Engineering, Kalasalingam Academy of Research and education, Krishnankoil, Tamil Nadu, India. He graduated B.E in Mechanical Engineering at AKCE in the year 2005, Graduated M.E in CAD/CAM at Mepco Schlenk Engg., college, Graduated Ph.D in Composite Materials in the year 2014 at Kalasalingam University, India. Also worked as Post Doc Research Associate in Precision Machining Lab, School of Mechanical Engineering, Yeungnam University, South Korea in the year 2017. He has very vast research experience in the field of composite materials with nearly 50 publications in various reputed SCI Journals and has nearly 40 publications in various International and National conferences.

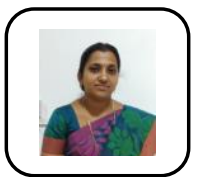

P. Sivaranjana working as assistant professor in the Department of Chemistry, School of Advanced Sciences, Kalasalingam Academy of Research and education. Her area of research is in biocomposites and she has very vast teaching experience of 13 years. 